\title{
Experimental and Computational Modelling of Flow Distribution
}

\author{
Dominika Babička Fialová ${ }^{1}$, Zdeněk Jegla ${ }^{1}$ \\ Institute of Process Engineering, Faculty of Mechanical Engineering, Brno University of Technology \\ Technická 2, 61669 Brno, Czech Republic \\ dominika.fialova@vutbr.cz; jegla@fme.vutbr.cz
}

\section{Extended Abstract}

In commercial software tools for process equipment design, the effect of maldistribution of working materials is usually neglected or simply flow distribution is considered ideally uniform. Analyses of this phenomenon and its negative consequences (namely increased local fouling rates, uneven extreme loading of the heat transfer surface, and mechanical failures) are mostly left to specialised computational fluid dynamics (CFD) models. The sophistication of current CFD simulations is at a level that many researchers rely on them as the only quality standard. However, the step of validating these numerical models using industrial data from the operation of the apparatuses or results of conducted experiments should not be neglected. A possible reason for preferring CFD simulations is that researchers often have little to do, as an operational or laboratory experiment is currently the most expensive option to investigate. Therefore, the same experiment results, the same industrial data are very often recycled in new publications. In addition to funding for the experiments, manufacturing of experimental devices or their reduced models is often a non-trivial problem for traditional manufacturing methods. Despite this, the new possibilities have emerged with the development of additive technologies. Due to this approach, it is quite easy to create complexly shaped models, e.g., flow distribution systems of heat transfer equipment.

The composite modelling system for the heat transfer analysis in equipment featuring complex designs [1], which is currently being developed, requires information on the distribution of fluid into tube bundles. Therefore, a fast and accurate-enough distribution model that would be easily implemented into the whole conception of the modelling system is sought. In this work, differential models based on the research of Bajura [2] and Bajura and Jones [3] are analysed. In order to validate the branch flow rates predicted by these mathematical models, the physical experiments are carried out using dividing manifold systems. Employed dividing headers (distributors) differs in a ratio of the outlet to the inlet area, i.e. one of the crucial parameters affecting flow distribution. The experimental distributors are additively manufactured by Fused Deposition Modelling technology from ABS material using 3D printer Trilab DeltiQ XXL by TriLAB Group s.r.o. (Brno, Czech Republic). Moreover, critical parts of the distribution systems, where different quantities would be measurable with much difficulty or not at all, are also inspected by the CFD simulations.

The results of the previous experiment [4] showed that both analysed mathematical models provide sufficiently accurate data on the distribution of the process fluid in a basic branched system. In this phase, it is necessary to identify a correct formulation of the mathematical model based on the data obtained from the tests of distributors with different geometry, in other words, the tests should determine the influence of the area ratio. The performance of each branched system is evaluated by means of relative standard deviation (RSD) from uniform flow distribution. In case of approximately same outlet and inlet areas (the area ratio around 1), the model [2] provides a more accurate prediction of flow rates as well as RSD, whereas the model [3] is more suitable for a system with the area ratio approx. 0,5.

In the future work, the experimental distributors, as well as their numerical models, will be used to verify the applicability of the suitable mathematical model to complete parallel flow systems and apparatuses with a higher number of tube rows.

This research has been supported by the project No. CZ.02.1.01/0.0/0.0/16_026/0008413 "Strategic partnership for environmental technologies and energy production", which has been co-funded by the Czech Ministry of Education, Youth and Sports within the EU Operational Programme Research, Development and Education.

\section{References}

[1] D. Fialová and Z. Jegla, "Analysis of Fired Equipment within the Framework of Low Cost Modelling Systems," Energies, vol. 12, no. 3, pp. 520:1-520:17, 2019. 
[2] R. A. Bajura, “A Model for Flow Distribution Manifolds," J. Eng. Power, vol. 93, no. 1, pp. 7-12, 1971.

[3] R. A. Bajura and E. H. Jones, "Flow Distribution Manifolds," J. Fluids Eng., vol. 98, no. 4, pp. 654-665, 1976.

[4] D. Babička Fialová and Z. Jegla, "Experimental validation of models for flow distribution in manifold systems," in Book of abstracts of the $66^{\text {th }}$ Conference of Chemical and Process Engineering CHISA, Seč, Czech Republic, 2019, p. 19. 